\title{
Prevalence of lipodystrophy and metabolic syndrome at the bertoua day hospital (east region of Cameroon)
}

\begin{abstract}
This cross-sectional study, carried out on a cohort of 568 HIV-infected patients followed at the Bertoua Day Hospital, aimed at assessing interventions used in the biomedical management of lipodystrophy and metabolic syndrome related to Antiretroviral therapy through the determination of their prevalence within this hospital. Patients had a minimum age of 18years old, a minimum duration of 2years antiretroviral therapy and all had given their informed consent to participate in the study. The mean duration of treatment for HIV patients with lipodystrophy cases was $68 \pm 9.2$ months ranged from 24 to 136 months and that for patients with metabolic syndrome cases was $46 \pm 8.5$ months ranged from 24 to 151 months $(\mathrm{P}$ value $=0.005)$. Lipodystrophy was observed with all the therapeutic protocols prescribed to HIV-infected patients in our study site. Lipodystrophy cases seen among HIV-infected patients using the $\mathrm{d} 4 \mathrm{~T}$ in their therapeutic protocol accounted for $41.11 \%$, while those seen among HIV-infected patients using the AZT in their therapeutic protocol accounted for $51.57 \%(\mathrm{P}<0.0001)$. Among HIV- infected patients using the NVP in their therapeutic protocol, we found $40.41 \%$ of lipodystrophy cases versus $28.23 \%$ of lipodystrophy cases among HIV-infected patients using the EFV in their therapeutic protocol $(\mathrm{P}$ value $=0.01)$. Lipodystrophy cases related to the use of protease inhibitors in the therapeutic protocol accounted for $31.36 \%$. The prevalence of metabolic syndrome cases was greatest among HIV-infected patients using protease inhibitors boosted by the ritonavir in their therapeutic protocol with $72.6 \%$ of cases $(\mathrm{P}$ value $=0.0003)$. The prevalence of metabolic syndrome cases determined in this study using the definition of the National Cholesterol Education Program Adult Treatment Panel III (NCEP-ATP III) was 21.83\% and that of lipodystrophy cases was $50.52 \%$ with $27.64 \%$ for lipoatrophy cases, $20.95 \%$ for lipohypertrophy cases and $1.93 \%$ for mixed syndrome cases $(\mathrm{P}$ value $=0.01)$. We also found $16.9 \%$ for lipodystrophy cases strongly associated with the metabolic syndrome among our HIV infected patients under HAART, with an Odds Ratio of 4.54 which was statistically significant with a confidence interval $(\mathrm{CI})$ between 3.45 and 6.87 to $95 \%$ ( $\mathrm{P}$ value $=0.03$ ) In other words, these HIV infected patients were 4.54 times more likely to have a metabolic syndrome in the presence of lipodystrophy than in its absence.
\end{abstract}

Keywords: lipodystrophy, metabolic syndrome, prevalence, haart, hiv infected patient
Volume 4 Issue 4 - 2017

\author{
Marcellin GuiaroNdoe,' Fak Moankong \\ Danielle Octavie,' Mbarawa Kofia Ibrahim \\ Marat, ${ }^{2}$ Antar Hamassi, ${ }^{3}$ Charles Fokunang \\ Ntungwen ${ }^{4,5}$ \\ 'Department of Biomedical Sciences, University of Ngaoundere, \\ Cameroon \\ ${ }^{2}$ Department of Medical Laboratory Sciences, University of \\ Buea, Cameroon \\ ${ }^{3}$ Faculty of Medicine and Pharmaceutical Sciences, University of \\ Douala, Cameroon \\ ${ }^{4}$ Faculty of Medicine and Biomedical Sciences, University of \\ Yaounde I, Cameroon 5 Faculty of Science, University of \\ Bamenda, Cameroon
}

Correspondence: Marcellin GuiaroNdoe, Department of Biomedical Sciences, Faculty of Science, University of Ngaoundere, Ngaoundere, Cameroon,

Email d.nokosore@yahoo.fr

Received: April 04, 2017 | Published: April 24, 2017
Abbreviations: CI, confidence interval; HAART, highly active antiretroviral therapies; HIV, human immunodeficiency virus; PI protease inhibitors; RTI, reverse transcriptase inhibitors; BMI, body mass index; NRTI, nucleotide reverse transcriptase inhibitors

\section{Introduction}

The use of highly active antiretroviral therapies (HAART) has reduced the mortality and morbidity associated with the Human Immunodeficiency Virus (HIV) infection. ${ }^{1}$ However, these treatments are associated with long term complications as the lipodystrophy syndrome, metabolic syndrome, diabetes mellitus, lactic acidosis etc. ${ }^{2}$ Among these complications, the lipodystrophy syndrome is often very remarkable. The unattractive nature of lipodystrophy can lead to self-stigmatization, stigmatization, marginalization and even reduced compliance in patients on treatment. ${ }^{3,4}$ The morphological and metabolic complications associated with the long-term use of HAART have been widely described in developed countries. In these countries, lipodystrophy syndrome has a prevalence ranging between 20 and $80 \%$ according to the studies. ${ }^{5}$ In the Aquitaine cohort in France, $38 \%$ of patients showed signs of lipodystrophy syndrome, including $16 \%$ for the lipoatrophy, $12 \%$ for the lipohypertrophy and $10 \%$ for the mixed syndrome. ${ }^{6}$ In sub-Saharan Africa, a Rwandan study estimated the prevalence of lipodystrophy syndrome at $34 \%{ }^{7}$ There is currently no precise definition of lipodystrophy syndrome, which in part explains the difference in prevalence between studies. Protease inhibitors (PI) and reverse transcriptase inhibitors (RTI), particularly the Stavudine, are the most implicated in the occurrence of morphological and metabolic abnormalities. ${ }^{8}$

The abnormal fat distribution in the body observed in the lipodystrophy syndrome is at the origin of the metabolic syndrome. Its prevalence, found in some studies made on it, is very dependent on the definition used. In France, this prevalence is estimated to $10 \%$ according to the American criteria of NCEP-ATP III (National Cholesterol Education Program Adult Treatment Panel III) from 2005. ${ }^{9}$ In Africa, Eholié in the Lipo-Afri study estimated the prevalence of the metabolic syndrome at $17.8 \% .{ }^{10}$ The occurrence of metabolic disorders among HIV-infected patients under HAART should consider their follow-up among HIV-infected patients because of the potential risk for cardiovascular, liver and diabetes complications. Hence the purpose of this study carried out on a cohort of HIV-infected patients followed at the Bertoua Day Hospital, in order to appreciate the interventions used in the biomedical follow-up for lipodystrophy and metabolic syndromes linked to HAART through the determination of their prevalence within that hospital. 


\section{Materials and methods}

\section{Study design, sampling and population}

We performed a cross-sectional stud with retrospective and prospective data collection from 05th November 2015 to 08th September 2016, on a cohort of 568 HIV-infected patients under HAART followed at the Bertoua Day Hospital. We performed a simple random sampling. Patient recruitment was carried out progressively at the time of their medical follow-up.

\section{Inclusion criteria and exclusion}

We included all patients infected with HIV-1 and/or 2, with at least two years old of antiretroviral therapy, with a minimum age of 18 years old and who have given their informed consent to participate in the study. We excluded from our study all patients with pregnancy; Renal, hepatic or cardiovascular diseases.

\section{Therapeutic protocol}

The therapeutic protocol of our HIV-infected patients consisted of $2 \mathrm{NRTI}+1 \mathrm{NNRTI}$ or $2 \mathrm{NRTI}+1 \mathrm{IP} / \mathrm{r}$ (Table $1 \&$ Figure 1$)$ and the mean duration for treatment of these patients was 4 years (48 months).

Table I Distribution of lipodystrophy types according to the therapeutic protocol

\begin{tabular}{lllll}
\hline $\begin{array}{l}\text { Therapeutic } \\
\text { Protocol }\end{array}$ & Lipoatrophy & Lipohypertrophy & $\begin{array}{l}\text { Mixed } \\
\text { Syndrome }\end{array}$ & Total \\
\hline d4T-3TC-NVP & $23(14.65 \%)$ & $18(15.13 \%)$ & $4(36.37 \%)$ & $45(15.68 \%)$ \\
AZT-3TC-NVP & $56(35.67 \%)$ & $12(10.08 \%)$ & $3(27.27 \%)$ & $71(24.73 \%)$ \\
AZT-3TC-EFV & $26(16.57 \%)$ & $14(11.77 \%)$ & $1(9.09 \%)$ & $41(14.3 \%)$ \\
d4T-3TC-EFV & $14(8.91 \%)$ & $26(21.85 \%)$ & 0 & $40(13.93 \%)$ \\
d4T 3TC LPV/r & $12(7.65 \%)$ & $19(15.96 \%)$ & $2(18.18 \%)$ & $33(11.5 \%)$ \\
AZT-3TC-LPV/r & $7(4.46 \%)$ & $16(13.44 \%)$ & $1(9.09 \%)$ & $24(8.36 \%)$ \\
AZT-3TC-IDV/r & $5(3.18 \%)$ & $7(5.89 \%)$ & 0 & $12(4.18 \%)$ \\
ABC-DDI-LPV/r & $8(5.09 \%)$ & $5(4.2 \%)$ & 0 & $13(4.54 \%)$ \\
ABC-TDF-LPV/r & $6(3.82 \%)$ & $2(1.68 \%)$ & 0 & $8(2.78 \%)$ \\
Total & $157(54.7 \%)$ & $119(41.47 \%)$ & $11(3.83 \%)$ & $287(100 \%)$ \\
\hline
\end{tabular}

The Percentages difference was statistically significant with a $P$ value $=0.0005$.

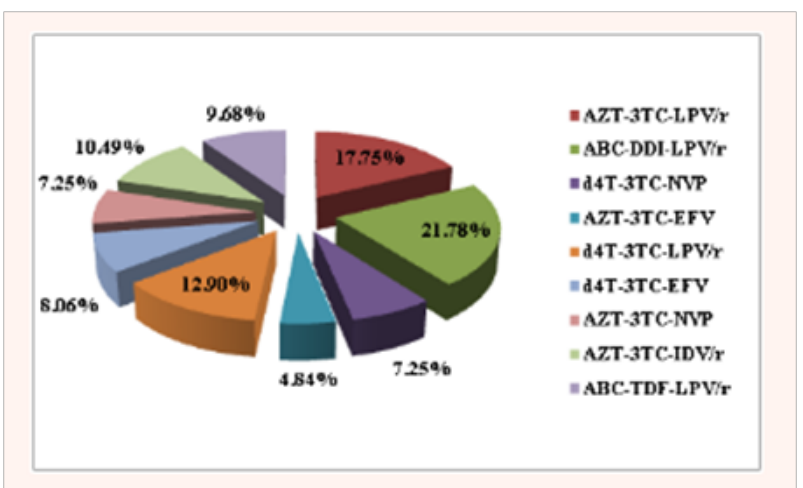

Figure I Prevalence of metabolic syndrome cases according to the therapeutic protocol.

\section{Collection of data}

It was done randomly in our place of study. We collected sociodemographic variables (age, sex), anthropometric variables (weight, height and waist), clinical variables (morphological variations and their perception since the beginning of HAART / lipodystrophy research), immunological variables and Virological (HIV type, CD4 count, viral load), Biochemical variables (total cholesterol, triglycerides, HDL-cholesterol, LDL-cholesterol, Blood sugar), Treatment-related variables (therapeutic protocol, duration of treatment). The data collection about the metabolic syndrome was done by focusing on the definition of the National Cholesterol Education Program Adult Treatment Panel III (NCEP-ATP III).

\section{Statistical analysis}

The data collected were analyzed using the version 2.13 of $\mathrm{R}$ software and Excel software. Chi-square and Ficher statistical tests were used to compare the different percentages of the variables studied with a significance threshold set at $5 \%$.

\section{Ethical Consideration}

Participation in the study was voluntary after being verbally informed about the objectives and constraints of the study. The confidentiality of participants was preserved throughout the study period and was not published.

\section{Results}

During our study period, we took a census of 568 participants (HIV-infected Patients) who were following their antiretroviral therapy at the Bertoua Day Hospital with a sex ratio of 0.67 in favor of the women who represented $59.85 \%$ of the participants in our study. Among these 568 participants, we identified 287 lipodystrophy cases, 124 cases for metabolic syndrome and 96 lipodystrophy cases associated with the metabolic syndrome with respective prevalences of $50.52 \%, 21.83 \%$ and $16.9 \%$. The prevalences of lipoatrophy cases, lipohypertrophy cases and mixed syndrome cases were $27.64 \%$, $20.95 \%$ and $1.93 \%$, respectively.

\section{The lipodystrophy syndrome}

\section{Demographic and anthropometric characteristics of HIV-infected patients with lipodystrophy}

Age: The mean age of HIV infected patients with lipodystrophy was $40 \pm 6.4$ years ranged from 18 to 72 years old. The age group of 50 years old and above had the highest percentages of lipoatrophy cases and lipohypertrophy cases with $53.5 \%$ and $43.7 \%$ respectively (Table 2).

Gender:The sex-ratio was 0.6 in favor of women which accounted for the highest percentage of lipodystrophy cases with $62.37 \%$ (Table 3 ).

Body mass index (BMI): mean of BMI for HIV-infected patients with lipodystrophy was $25 \pm 8.6 \mathrm{~kg} / \mathrm{m}^{2}$. The lipoatrophy cases were more represented among patients with $\mathrm{BMI} \leq 18.5 \mathrm{~kg} / \mathrm{m} 2$ with a rate of $47.13 \%$ (Table 4 ).

\section{Characteristics related to HIV infection}

Therapeutic protocol: Lipodystrophy cases observed among HIVinfected patients using a therapeutic protocol including the d4T accounted for $41.11 \%$, while those observed among HIV-infected patients using a therapeutic protocol including the AZT accounted for $51.57 \%(\mathrm{P}<0.0001)$. Among HIV-infected patients using the NVP in their therapeutic protocol, we found $40.41 \%$ of lipodystrophy cases versus $28.23 \%$ of lipodystrophy cases among patients using the EFV in their therapeutic protocol ( $\mathrm{P}$ value $=0.01$ ). The cases of lipodystrophy related to the use of protease inhibitors in the therapeutic regimen accounted for $31.36 \%$ (Table 1).

The duration of HAART: The mean duration of HAART was $68 \pm 9.2$ 
months ranged from 24 to 136 months. We also noticed cohesion between the duration of antiretroviral therapy and the increasing percentages of lipodystrophy cases recorded during our study period (Table 5).
The Percentages difference was statistically significant with a $\mathrm{P}$ value $=0.001$.

Table 2 Distribution of HIV-infected patients with lipodystrophy according to the age group and type of lipodystrophy

\begin{tabular}{lllll}
\hline Age Group (In Year Old) & Lipoatrophy & Lipohypertrophy & Mixed Syndrome & Total \\
\hline 18 to 30 & $5(3.19 \%)$ & $8(6.72 \%)$ & 0 & $13(4.53 \%)$ \\
30 to 40 & $22(14.01 \%)$ & $28(23.53 \%)$ & $2(18.18 \%)$ & $52(18.11 \%)$ \\
40 to 50 & $46(29.3 \%)$ & $31(26.05 \%)$ & $6(54.55 \%)$ & $83(28.93 \%)$ \\
50 and above & $84(53.5 \%)$ & $52(43.7 \%)$ & $3(27.27 \%)$ & $139(48.43 \%)$ \\
Total & $157(54.7 \%)$ & $119(41.47 \%)$ & $11(3.83 \%)$ & $287(100 \%)$ \\
\hline
\end{tabular}

The percentages difference was statistically significant with a $P$ value $=15.74 \times 10^{-5}$.

Table 3 Distribution of HIV-infected Patients with lipodystrophy according to the gender and type of lipodystrophy

\begin{tabular}{lllll}
\hline Gender & Lipoatrophy & Lipohypertrophy & Mixed Syndrome & Total \\
\hline Male & $108(37.63 \%)$ & $65(41.4 \%)$ & $40(33.6 \%)$ & $3(27.27 \%)$ \\
Female & $179(62.37 \%)$ & $92(58.6 \%)$ & $79(66.4 \%)$ & $8(72.73 \%)$ \\
Total & $287(100 \%)$ & $157(54.7 \%)$ & $119(41.47 \%)$ & $11(3.83 \%)$ \\
\hline
\end{tabular}

The percentages difference was statistically significant with a $P$ value $=0.03$.

Table 4 Distribution of HIV-infected patients with lipodystrophy according to BMI and type of lipodystrophy

\begin{tabular}{lllll}
\hline BMI (kg/m2) & Lipoatrophy & Lipohypertrophy & Mixed Syndrome & Total \\
\hline$\leq 18.5$ & $74(47.13 \%)$ & 0 & 0 & $74(25.79 \%)$ \\
{$[18.5-25]$} & $44(28.02 \%)$ & $26(21.85 \%)$ & $\mathrm{I}(9.09 \%)$ & $71(24.73 \%)$ \\
{$[25-30]$} & $29(18.48 \%)$ & $55(46.22 \%)$ & $3(27.28 \%)$ & $87(30.31 \%)$ \\
$>30$ & $10(6.37 \%)$ & $38(31.93 \%)$ & $7(63.63 \%)$ & $55(19.17 \%)$ \\
Total & $157(54.7 \%)$ & $119(41.47 \%)$ & $1 \mathrm{I}(3.83 \%)$ & $287(100 \%)$ \\
\hline
\end{tabular}

The percentages difference was statistically significant with a P.value $=1.87 \mathrm{e}^{-08}$.

Table 5 Distribution of HIV-infected patients with lipodystrophy according to the duration of HAART and type of lipodystrophy

\begin{tabular}{lllll}
\hline HAART Duration (In months) & Lipoatrophy & Lipohypertrophy & Mixed Syndrome & Total \\
\hline$[24-36]$ & $9(5.73 \%)$ & $3(2.53 \%)$ & 0 & $12(4.18 \%)$ \\
{$[36-48]$} & $20(12.74 \%)$ & $7(5.88 \%)$ & $\mathrm{I}(9.09 \%)$ & $28(9.76 \%)$ \\
{$[48-60]$} & $26(16.57 \%)$ & $21(17.65 \%$ & $\mathrm{I}(9.09 \%)$ & $48(16.72 \%)$ \\
{$[60-72]$} & $29(18.47 \%)$ & $23(19.32 \%)$ & $3(27.28 \%)$ & $55(19.16 \%)$ \\
{$[72-84]$} & $31(19.74 \%)$ & $29(24.37 \%)$ & $2(18.18 \%)$ & $62(21.6 \%)$ \\
$\geq 84$ & $42(26.75 \%)$ & $36(30.25 \%)$ & $4(36.36 \%)$ & $82(28.58 \%)$ \\
Total & $157(54.7 \%)$ & $119(41.47 \%)$ & $11(3.83 \%)$ & $287(100 \%)$ \\
\hline
\end{tabular}

Table 6 Prevalence of metabolic syndrome types diagnosed in our study population

\begin{tabular}{llc}
\hline Type of Metabolic Syndrome & Percentages & Prevalences \\
\hline Abdominal Obesity + Hyperglycemia + HypoHDLemia & $15.32 \%$ & $3.35 \%$ \\
Abdominal Obesity + HypoHDLemia + Hypertriglyceridaemia & $17.75 \%$ & $3.88 \%$ \\
Abdominal Obesity + Hypertriglyceridaemia + hypertension & $12.90 \%$ & $2.81 \%$ \\
Abdominal Obesity + Hyperglycemia + HypoHDLemia + hypertension & $9.68 \%$ & $2.11 \%$ \\
Abdominal Obesity + HypoHDLemia + Hypertriglyceridaemia + hypertension & $12.09 \%$ & $2.64 \%$ \\
Abdominal Obesity + Hypertriglyceridaemia + hypertension & $10.48 \%$ & $2.29 \%$ \\
HypoHDLemia + Hypertriglyceridaemia + Hyperglycemia & $7.26 \%$ & $1.59 \%$ \\
HypoHDLemia + Hyperglycaemia + hypertension & $4.84 \%$ & $1.05 \%$ \\
HypoHDLemia + hypertriglyceridemia + hypertension & $2.42 \%$ & $0.53 \%$ \\
Hypertriglyceridaemia + Hyperglycaemia + hypertension & $5.64 \%$ & $1.23 \%$ \\
HypoHDLémie + Hypertriglyceridaemia + Hyperglycemia + hypertension & $1.62 \%$ & $0.35 \%$ \\
Total & $100 \%$ & $21.83 \%$ \\
\hline
\end{tabular}

The difference in rates was not statistically significant with a P.value $=0.36$. 
Table 7 Distribution of HIV-infected patients according to the lipodystrophy associated with the metabolic syndrome

\begin{tabular}{|c|c|c|c|c|}
\hline \multirow[t]{2}{*}{ Metabolic Syndrome } & \multirow[t]{2}{*}{$\begin{array}{l}\text { OR (Cl to } \\
95 \%)\end{array}$} & $P$ value & & \\
\hline & & Yes & \multicolumn{2}{|l|}{ No } \\
\hline \multirow{2}{*}{ Lipodystrophy } & $287(50.52 \%$ & $\begin{array}{l}96 \\
(33.4 \%)\end{array}$ & $\begin{array}{l}191 \\
(66.6 \%)\end{array}$ & \multirow{2}{*}{$\begin{array}{l}4.54(3.45-0.03 \\
6.87)\end{array}$} \\
\hline & $28 I(49.48 \%$ & $\begin{array}{l}28 \\
(9.96 \%)\end{array}$ & $\begin{array}{l}253 \\
(90.04 \%)\end{array}$ & \\
\hline
\end{tabular}

$\mathrm{Cl}$, confidence interval; OR, odds ratio.

Table 8 Distribution of HIV-infected patients according to the types of lipodystrophy associated with the mixed syndrome

\section{Mixed Syndrome}

\begin{tabular}{|c|c|c|c|c|c|c|}
\hline \multicolumn{2}{|c|}{ Types of lipodystrophy } & \multicolumn{3}{|c|}{ Yes No } & \multirow{2}{*}{$\begin{array}{l}\text { OR ( } \mathrm{Cl} \text { to } 95 \%) \\
2.09\end{array}$} & \multirow{2}{*}{ P Value } \\
\hline & Yes & 119 & 64 & 55 & & \\
\hline стропурет сториу & No & 168 & 60 & 108 & $(1.06-3.58)$ & 0.01 \\
\hline \multirow{2}{*}{ Lipoatrophy } & Yes & 157 & 23 & 134 & 0.04 & \multirow{2}{*}{0.001} \\
\hline & No & 130 & 101 & 29 & $(-0.9-1.53)$ & \\
\hline \multirow{2}{*}{ Mixed Syndrome } & Yes & II & 9 & 2 & 6.49 & \multirow{2}{*}{0.04} \\
\hline & No & 276 & 113 & 163 & $(5.46-7.98)$ & \\
\hline
\end{tabular}

$\mathrm{Cl}$, confidence interval; $\mathrm{OR}$, odds ratio.

\section{Morphological modifications}

Lipodystrophy localization: In a same HIV-infected patient we could notice several lipodystrophy localizations. But, the abdomen was the most frequent localization with $43.21 \%$ of cases, followed by the buttocks with $22.99 \%$. The Percentages difference was statistically significant with a $\mathrm{P}$ value $=0.05$ (Figure 2).

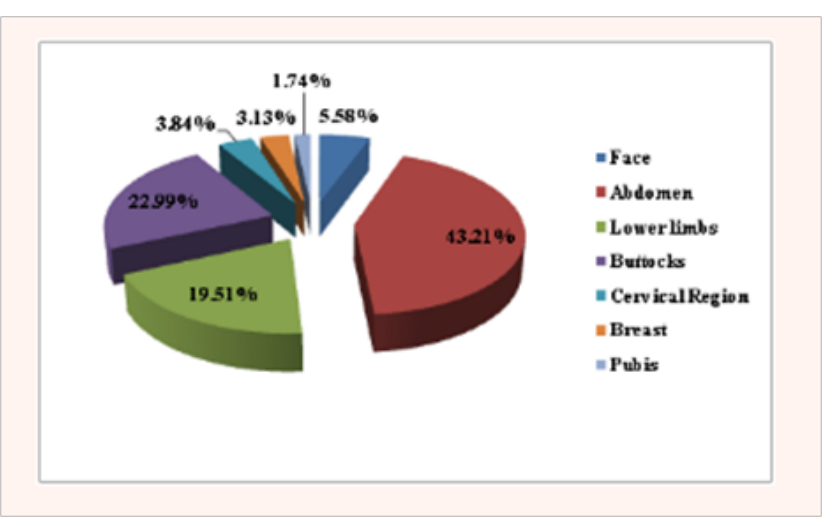

Figure 2 Distribution of HIV-infected patients according to the lipodystrophy localization.

\section{The metabolic syndrome}

Demographic and anthropometric characteristics of Metabolic Syndrome

Age: The prevalence of metabolic syndrome cases increased with age. The mean age was $42 \pm 8.6$ years old ranged from 18 to 72 years old. The age group of 50 and above was the most represented with $41.51 \%$ metabolic syndrome cases. The percentages difference was statistically significant with a $\mathrm{P}$ value $=0.04$ (Figure 3 ).

Gender: The sex ratio was 0.58 in favor of women which accounted for the highest percentage of metabolic syndrome cases with $63 \%$
(Figure 4). The percentage difference was statistically significant with a $P$ value $=0.01$

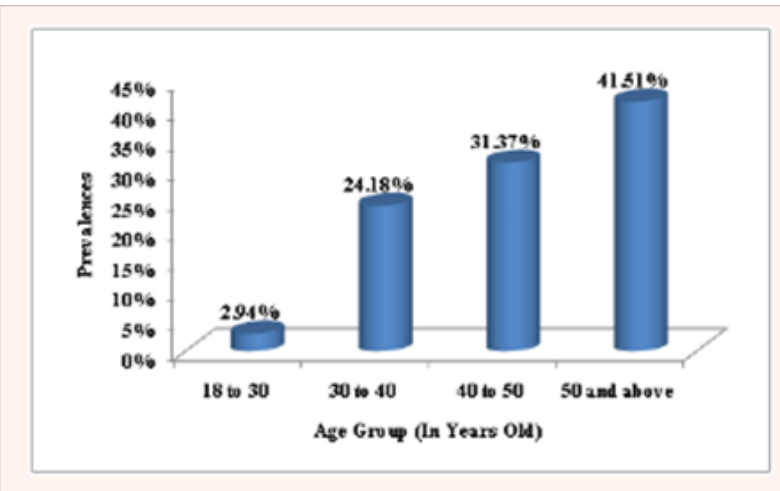

Figure 3 Prevalence of metabolic syndrome according to the age group.

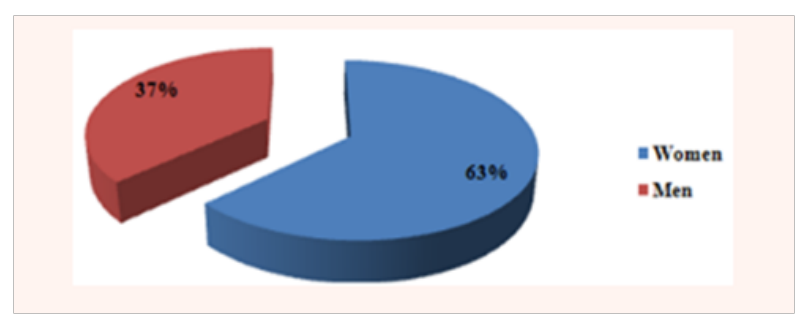

Figure 4 Prevalence of metabolic syndrome cases according to the gender.

Body mass index (BMI) : The mean of BMI was $28.32 \pm 5.7 \mathrm{Kg} / \mathrm{m}^{2}$. The prevalence of metabolic syndrome cases was higher in the BMI range from 25 to $30 \mathrm{Kg} / \mathrm{m} 2$ with $48.36 \%$ (Figure 5). The percentages difference was not statistically significant with a $\mathrm{P}$ value $=0.54$.

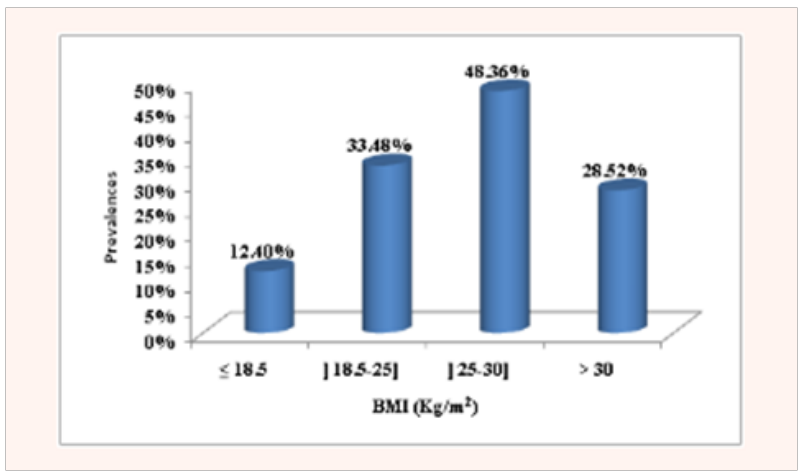

Figure 5 Prevalence of metabolic syndrome cases according to the Body Mass Index.

\section{Therapeutic aspect of the metabolic syndrome}

Therapeutic protocol: The prevalence of metabolic syndrome cases was higher among HIV-infected patients using the protease inhibitors boosted by the ritonavir (Figure 1). The percentage difference was statistically significant with a $\mathrm{P}$ value $=0.0003$.

The duration of HAART: The prevalence of metabolic syndrome cases also increased with the HAART duration. The mean HAART duration was $46 \pm 8.5$ months ranged from 24 to 151 months. The prevalence of metabolic syndrome cases was higher among HIVinfected patients with HAART duration $\geq 84$ months (Figure 6). The percentages difference was statistically significant with a $\mathrm{P}$ value $=0.005$

Type of Metabolic Syndrome: The metabolic syndrome with 
abdominal obesity was the most represented among our HIV-infected patients with $78.2 \%$ and a general prevalence of $17.07 \%$ (Table 6).

\section{Lipodystrophy and metabolic syndrome}

Among the 287 HIV-infected patients with lipodystrophy, 96(33.4\%) had a metabolic syndrome (Table 7$)$. We found a strong association between lipodystrophy and metabolic syndrome in our HIV-infected patients with an Odds Ratio $=4.54$ which was statistically significant with a confidence interval (CI) between 3.45 and 6.87 to $95 \%$ and $\mathrm{P}$ value $=0.03$.

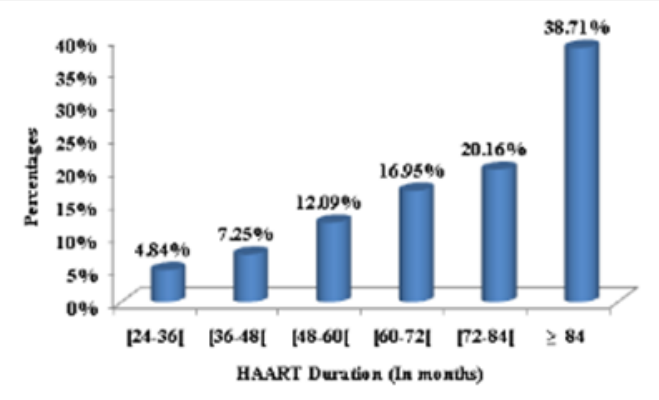

Figure 6 Prevalence of metabolic syndrome cases according to HAART duration.

Types of lipodystrophy associated with the metabolic syndrome : By focusing our attention on types of lipodystrophy, we found that lipohypertrophy and mixed syndrome were strongly associated with the metabolic syndrome with an Odds Ratio of 2.09 and 6.49 respectively. These Odds Ratio were all statistically significant (Table 8).

\section{Discussion}

\section{Prevalence}

Our study reports a prevalence of $50.52 \%$ of lipodystrophy cases; with $27.64 \%$ of lipoatrophy cases, $20.95 \%$ of lipohypertrophy cases and $1.93 \%$ of mixed syndrome cases. This prevalence of lipodystrophy cases varies from one study to another. In Rwanda, Mutimura found a prevalence of $34 \% .^{7}$ Mercier in Senegal ${ }^{11}$ and Zannou in Benin ${ }^{12}$ found prevalences of $31.1 \%$ and $30 \%$, respectively. In the Aquitaine cohort in France, ${ }^{13} 38 \%$ of HIV-infected patients showed lipodystrophy signs, including $16 \%$ for lipoatrophy cases, $12 \%$ for lipohypertrophy cases and $10 \%$ for mixed syndrome cases. Pujari in India [8] found a prevalence of $46.1 \%$. Miller in Australia found a prevalence of $53 \%$ for lipodystrophy cases, with $31 \%$ for lipoatrophy cases, $14 \%$ for lipohypertrophy and $55 \%$ for mixed syndrome. ${ }^{14}$ This prevalence difference is partly due to the fact that there is currently no consensual definition of lipodystrophy but also to the variety of therapeutic protocols used and the duration of exposure to antiretroviral drugs. ${ }^{15}$

The prevalence of metabolic syndrome cases in our study was $21.83 \%$ according to the definition of NCEP-ATP III. It is higher than that found in studies made by Sawadogo in Burkina Faso, ${ }^{16}$ Jerico in Spain ${ }^{17}$ and Biron in France, ${ }^{18}$ who found prevalences of $14 \%, 17 \%$ and $19.9 \%$ respectively with the same definition of the metabolic syndrome used in our study. Eholié in the Lipo-Afri study reported a prevalence of $17.8 \%$ with the IDF definition. ${ }^{10}$ Indeed, the prevalence of the metabolic syndrome is highly dependent on the definition used. However, the difference in the results obtained with the same definition criteria in our study (NCEP-ATP III) also seems to be related to the nature of the therapeutic protocols used and the duration of exposure to antiretroviral drugs.

We also found $16.9 \%$ of lipodystrophy cases strongly associated with the metabolic syndrome among our HIV patients under HAART, with an Odds Ratio of 4.54 which was statistically significant and a confidence interval (CI) between 3.45 and 6.87 to $95 \%$. In other words, these HIV-infected patients were 4.54 times more likely to have a metabolic syndrome in the presence of lipodystrophy than in its absence (Table 7). By focusing our attention on the types of lipodystrophy, we found that lipohypertrophy and mixed syndrome were strongly associated with the metabolic syndrome with statistically significant Odds Ratio of 2.09 and 6.49 respectively (Table 8). Under HAART, our HIV-infected patients with lipohypertrophy were 2.09 times more likely to have a metabolic syndrome and those with mixed syndrome were 6.49 times more likely to have metabolic syndrome. This observation was also made by Thiébaut and Zannou in their study with the definition of the IDF., ${ }^{6,12}$ The literature reports that dyslipidemias are associated but not always with lipodystrophy among HIV-infected patients under HAART. $9,2,15,19$

\section{Age}

The mean age of HIV-infected patients with lipodystrophy was $40 \pm 6.4$ years old ranged from 18 to 72 years old. The age group of 50 years and above had the highest percentages of lipoatrophy cases and lipohypertrophy cases with $53.5 \%$ and $43.7 \%$ respectively (Table 2). Older age would be predisposing to lipodystrophy among the HIVinfected patient under HAART. This finding is similar to the results of many other studies about lipodstrophy among HIV-infected patients under HAART. ${ }^{6,7,8,12}$

The mean age of HIV-infected patients with the metabolic syndrome cases under HAART was $42 \pm 8.6$ years old ranged from 18 to 72 years old. The age group of 50 and above was also the most represented with $41.51 \%$ of metabolic syndrome cases (Figure 3). This finding in our study reflects the fact that the prevalence of the metabolic syndrome increases with age. Eholié and Jerico made the same observation in their studies. ${ }^{10,17}$ Ageing would be a predisposing factor to the metabolic syndrome among HIV-infected patients under HAART. Studies have shown that HIV-infected patients have the same complications related to the ageing (dyslipidemia, diabetes and insulin resistance) $10-15$ years earlier than the general population. ${ }^{19,20}$

\section{Sex}

The sex-ratio was 0.67 in favor of women who represented $59.85 \%$ of participants in our study. This could be explained by the fact that the follow-up for the antiretroviral therapy is more regular among women than among men because of their body physiology. This finding was also made by Marcellin in his study about the Nutritional State Impact on Liver Detoxification Function among Infected Patients with HIV under Antiretroviral Drugs ${ }^{21,22}$ in the same study site. This difference in body physiology could also explain the large percentage of lipodystrophy cases $(62.37 \%)$ found among women in our study (Table 3 ). This finding was confirmed by Galli in his study about the Gender differences in antiretroviral drug related adipose tissue alterations. ${ }^{23}$

The prevalence of the metabolic syndrome was also higher in women with $63 \%$ versus $37 \%$ in men (Figure 4 ). This result is similar to the one reported in the studies made by Zannou and Samaras. ${ }^{12,24}$ This result is also confirmed in Galli's study, which reports that hypertrophy of visceral adipose tissue is more common among women than among men. ${ }^{25}$ 


\section{The body mass index (BMI)}

The mean of BMI among HIV-infected patients with lipodystrophy was $25 \pm 8.6 \mathrm{~kg} / \mathrm{m}^{2}$. We found that lipoatrophy was more represented among HIV-infected patients with BMI $\leq 18.5 \mathrm{~kg} / \mathrm{m}^{2}$ with $47.13 \%$ (Table 4). This finding is somewhat distant from those made by Griensven in Rwanda in his study about the prevalence of lipoatrophy among HIV-infected patients under 1st line of the antiretroviral therapy and by Thiébaut in the Aquitaine cohort in France. ${ }^{6,25}$

The hypothesis of changes in the volume of body fat (loss or accumulation) that influence the weight and the BMI value can give an explanation for this finding made in our study. Young in his study about the Body fat changes among antiretroviral-naïve patients on PI and NNRTI-based HAART in the Swiss HIV Cohort Study, showed the relationship between volume changes of body fat and BMI. ${ }^{26}$

The average of BMI among HIV-infected patients with the metabolic syndrome was $28.32 \pm 5.7 \mathrm{Kg} / \mathrm{m}^{2}$. The prevalence of these metabolic syndrome cases was higher in the BMI ranged from 26 to $30 \mathrm{Kg} / \mathrm{m} 2$ with $48.36 \%$ (Figure 5). Our results corroborate with those found in studies made by Eholié, Biron and Jerico..$^{10,17,18}$ The hypertrophy of the adipose tissue or the accumulation of fat in particular visceral being a key element in the definition of the metabolic syndrome, according to the criteria of the NCEP-ATP III could still explain our results.

\section{The therapeutic protocol}

Lipodystrophy was observed with all therapeutic protocols prescribed in our study site for HIV-infected patients. AZT and d4T belonging to the class of NRTI, incriminated in the occurrence of lipodystrophy, ${ }^{27}$ were found in the therapeutic protocol of patients with lipodystrophy in $51.57 \%$ and $41.11 \%$ of cases respectively $(\mathrm{P}<0.0001)$. Lipoatrophy cases were more represented among our HIV-infected patients treated with NRTI (d4T or AZT) with $75.8 \%$ compared to $58.85 \%$ of lipohypertrophy cases (Table 1). This percentages difference was statistically significant with a $\mathrm{P}$ value $=0.01$ and this finding is justified in the literature review which highlights the toxic effect of nucleotide reverse transcriptase inhibitors (NRTI) on the mitochondrial DNA $\gamma$ polymerase which Inhibits the functioning of the cellular respiratory chain and inhibits the oxidation pathways of fatty acids (dysfunction of adipose cells), hence the appearance of subcutaneous lipoatrophy. ${ }^{28,29}$ Griensven in his study about the prevalence of lipoatrophy made the same observation as us; $31.4 \%$ of HIV-infected patients with d4T had lipoatrophy $(\mathrm{p}=0.019) .{ }^{25}$ Similarly, Mercier in his study showed that exposure to d4T greater than 6 months was significantly associated with lipodystrophy $(\mathrm{p}=0.004)$. Pujari in his study found a significant association between the use of $\mathrm{d} 4 \mathrm{~T}$ and the occurrence of lipoatrophy $(\mathrm{p}=0.04)$ and no association between the use of therapeutic protocol such as AZT-3TCNVP, D4T-3TC-NVP and the occurrence of lipodystrophy. ${ }^{8}$

The cases of lipodystrophy related to the use of protease inhibitors in the therapeutic protocol accounted for $31.36 \%$ (Table 1). Our proportion is somewhat lower than the $37 \%$ reported by Thiébaut ${ }^{6}$ and the $43.9 \%$ reported by Eholié in the Lipo-Afri study. ${ }^{10}$ Studies have shown a significant association between the use of protease inhibitors and lipodystrophy among HIV-infected patients. ${ }^{6,14,30}$ Indeed, the relationship between protease inhibitors and lipodystrophy is known. Protease inhibitors would lead to oxidative stress and hypertrophy of adipose tissue..$^{31,32}$

Among HIV-infected patients using the NVP in their therapeutic protocol, we found $40.41 \%$ of lipodystrophy cases versus $28.23 \%$ of lipodystrophy cases among HIV-infected patients using the EFV in their therapeutic protocol with $\mathrm{P}$ value $=0.01$ (Table 1). This finding is similar to the one made by Mutimura who found in his study that the use of the EFV was associated with a lower prevalence of lipodystrophy cases than the NVP, that is $15.4 \%$. $^{7}$ Indeed, previous studies that have addressed the issue of NNRTI and their relationship to lipodystrophy have concluded that NNRTI have more favorable effects on the redistribution of fat mass. ${ }^{11,33}$ Concerning the metabolic syndrome, its prevalence was higher among HIV-infected patients using protease inhibitors boosted by the ritonavir with $82.28 \%$ of cases (Figure 6). Our results are similar to those found by Thiébaut, Eholiée \& Jerico. ${ }^{6,10,17,34}$ Indeed, it is recognized that the majority of Protease inhibitors disrupt lipid parameters and lead to elevated triglycerides, total cholesterol and LDL-cholesterol, as well as an increase of the blood sugar level and insulinaemia, but to different degrees. ${ }^{2,15,35,36}$

\section{Duration of exposure to antiretroviral therapy}

The mean duration of the antiretroviral therapy for HIV-infected patients with lipodystrophy was $68 \pm 9.2$ months ranged from 24 to 136 months. We also found a cohesion between the duration of the antiretroviral therapy and the increasing percentages of lipodystrophy cases recorded during our study period (Table 5). Our finding is similar to that found in a large number of lipodystrophy study that also found that long exposure to HAART was associated with lipodystrophy ${ }^{6,14,25,30,36,37}$ The finding evoked in our study could be explained in the literature review which reports that the toxicity of some ARV drugs induces a mitochondrial dysfunction and an oxidative stress that are at the origin of the occurrence of the lipodystrophiy syndrome. ${ }^{31,37}$ As a result, long exposure to these molecules would promote the occurrence of this dysfunction.

The prevalence of the metabolic syndrome cases also increased with duration of the antiretroviral therapy. The mean duration of the antiretroviral therapy for our HIV-infected patients under HAART was $46 \pm 8.5$ months ranged from 24 to 151 months. The prevalence of metabolic syndrome cases was higher among patients with therapy duration $\geq 84$ months (Figure 6). Our finding is similar to the one made by Thiébaut in the Aquitaine cohort in France despite the difference in duration of exposure to antiretroviral therapy. ${ }^{6}$ Our results can be explained with the role played by ARV drugs in the occurrence of metabolic disorders that appear in the long term during the exposure period.

\section{Localization of the lipodystrophy}

In a same HIV-infected patient we could notice several localizations of the lipodystrophy. But the abdomen was the most frequent localization with $43.21 \%$ of cases, followed by the buttocks with $22.99 \%$. (Figure 2). Our finding, despite the difference in percentage, is similar to those made by Mutimura and Thiébaut, who also found that the abdomen was most affected by lipodystrophy compared to other parts of the body, respectively in $84 \%$ and $20.7 \%$ of cases. ${ }^{6,7}$ The high consumption of alcohol and fatty food found among our HIV-infected patients under HAART could justify this finding made in our study.

\section{Limitations of the study}

Scientific honesty allows us to admit that this study was not perfect, and thus, its limits should be precised. The prevalences found in our study don't really extend to the whole of Bertoua city (East Region of Cameroon), because of the difficulties of regular followup for HIV-infected patients under HAART namely: loss of view, transfer, abandonment of antiretroviral therapy and deaths. 


\section{Conclusion}

Our study, despite its limitations, reports a prevalence of $27.64 \%$ of lipoatrophy cases, $20.95 \%$ of lipohypertrophy cases and $1.93 \%$ of mixed syndrome cases with an overall prevalence of $50.52 \%$ of lipodystrophy cases. The prevalences of metabolic syndrome cases and lipodystrophy cases associated with the metabolic syndrome were $21.83 \%$ and $16.9 \%$ respectively. The management of these pathological entities (lipodystrophy and metabolic syndrome) has not yet been officially recognized in Cameroon and raises several problems affecting their diagnosis and treatment. The Ministry of Public Health should regularly provide equipments for the biological and medico-surgical management of the lipodystrophy; Facilitate the access of HIV-infected patients to lipid-lowering agents such as Pravastatin, Rosuvastatin and fibrates; Making more accessible the less toxic molecules such as FTC, ABC and TDF recommended in first-line treatment according to the new WHO recommendations as well as the proteases inhibitors considered to be less deleterious (Atazanavir, Saquinavir etc.) more accessible, and to appoint also in the care structures, the dieticians who will act as adviser for the HIVinfected patients about the respect of the standard hygiene-dietetic measures with regard to the lipodystrophy and metabolic syndrome. The scientific community should also carry out further investigations about the metabolic syndrome resulting from the abnormal distribution of fat in the body in order to know if all its components are known, such as the uricemia, which is an element totally ignored in all current definitions that have been the subject of an international consensus.

\section{Authorship contribution}

All authors contributed to the designing, preparation, editing, and final review of the manuscript.

\section{Acknowledgements}

Authors thank the collaborators of their respective institutions for the comments on the manuscript.

\section{References}

1. Morcroft A, Ledergerber B, Katlama C, et al. Decline in the AIDS and death rates in the EuroSIDA study: an observational study. Lancet. 2003;362(9377):22-29.

2. Carr A, Samras K, Burton S, et al. A syndrome of peripheral lipodystrophy, hyperlipidemia, and insulin resistance in patients receiving HIV protease inhibitors. AIDS. 1998;12(7):51-58.

3. Fernandes APM, Sanches RS, Mill J, et al. Lipodystrophy syndrome associated with antiretroviral therapy in HIV patients: considerations for psychosocial aspects. Rev Lat Am Enfermagem. 2007;15(5):1041-1045.

4. Power R, Tate H L, Mc Gill SM, et al. A qualitative study of the psychosocial implications of lipodystrophy syndrome on HIV positive individuals. Sex Transm Infect. 2003;79(2):137-141.

5. Grinspoon S, Carr A. Cardiovascular risk and body-fat abnomalities in HIV-infected adults. N Engl J Med. 2005;352(1): 48-62.

6. Thiébaut R, Daucourt V, Mercié P, et al. Lipodystrophy, Metabolic Disorders, and Human Immunodeficiency Virus Infection: Aquitaine Cohort. Clin Infect Dis. 2000;31(6):1482-1487.

7. Mutimura E, Stewart A, Rheeder P, et al. Metabolic function and the prevalence of lipodystrophy in a population of HIV-infected African subjects receiving highly active antiretroviral therapy. J Acquir Immune Decfic Syndr. 2007;46(4):451-455

8. Pujari SN, Dravid A, Naik E, et al. Lipodystrophy and dislipideamia among patients taking first line world health organization-recommended highly active antiretroviral therapy regimens in western India. J Acquir Immune Defic Syndr. 2005; 39(2):199-202.

9. Capeau J, Valantin MA. Altérations et al.métaboliques et vieillissement prématuré au cours de l'infection VIH et en réponse aux traitements antirétroviraux. In: VIH de Girard PM, et al. Doin Paris, France. $2011 ; 544-548$

10. Eholié SP, Lacombe K, Krain A, et al. Incidence des lipodystrophies, des anomalies métaboliques et évaluation du risque cardiovasculaire dans une cohorte. de patients originaires d'Afrique subsaharienne sous multithérapie antirétroviral. Journées Nationales d'Infectiologie Dijon. 2007.

11. Mauss S, Corzillius M, Wolf E, et al. Risk factors for HIV-associated lipodystrophy syndrome in a closed cohort of patients after 3 years of antiretroviral treatment. HIV Med. 2002;3(1):49-55.

12. Zannou DM, Denoeud L, Lacombe K, et al. Incidence of lipodystrophy and metabolic disorders in patients starting non-nucleoside reverse transcriptase inhibitors in Benin. Antivir Ther. 2009;14(3):371-380.

13. Capeau J, Valantin MA. Syndrome lipodystrophique au cours des traitements antirétroviraux. In: VIH de Girard PM, et al. (Eds.), Doin Paris, France. 2011:525-537.

14. Miller J, Carr A, Emery S, et al. HIV lipodystrophy: prevalence, severity and correlates of risk in Australia 2003 British HIV Association. HIV Med. 2003;4(3):293-301.

15. Grinspoon S, Carr A (2005) Cardiovascular risk and body-fat abnomalities in HIV-infected adults. N Engl J Med. 2003;352:48-62.

16. Sawadogo KCS. Facteurs de risque cardiovasculaire chez les personnes vivant avec le VIH à l'ère des combinaisons d'antirétroviraux à Ouagadougou. UFR Sciences de la Santé, Thèse Med. 2009;59-66.

17. Jericó C, Knobel H, Montero M, et al. Metabolic syndrome among HIV-infected patients: prevalence, characteristics, and related factors. Diabetes care. 2005;28(1):132-137.

18. Biron C, Bobin DC, Leport C, et al. Syndrome métabolique chez les patients VIH+ sous HAART depuis 1 an à 4 ans:étude SYMET. Elsev Masson. 2008;38:170-172.

19. Caron-debarle M, Lagathu C, Boccara F, et al. HIV-associated lipodystrophie: from fat injury to premature aging. Trends Mol Med. 2010;16(5):218-229.

20. Deeks SG, Phillips AN. HIV infection, antiretroviral treatment, ageing, and non-AIDS related morbidity. BMJ. 2009;339: a3172.

21. Effors RB, Fletcher CV, Gebo K, et al. Aging and infectious diseases. Workshop on HIV infection and aging: what are known and future research directions? Clin Infect Dis. 2008;47(4):542-553

22. Marcellin Guiaro Ndoe, Armel Herve Nwabo Kamdje, Charles Fokunang Ntungwen, et al. Nutritional State Impact on Liver Detoxification Function in Patients Infected with HIV under Antiretroviral Drugs at the Bertoua Day Hospital (Cameroon). Journal of Diseases and Medicinal Plants. 2015;1(2):37-41.

23. Galli M, Veglia F, Angarano G, et al. Gender differences in antiretroviral drug related adipose tissue alterations: women are at higher risk than men and develop particular lipodystrophy patterns. J Acquir Immune Defic Syndr. 2003;34(1):58-61.

24. Griensven VJ, De Naeyer I, Mushi T, et al. High prevalence of lipoatrophy among patients on stavudine-containing first-line antiretroviral therapy regimens in Rwanda. Trans R Soc Trop Med Hyg. 2007;101(8):793-798.

25. Samaras K, Wand H, Law M, et al. Prevalence of metabolic syndrome in HIV-infected patients receiving in highly active antiretroviral therapy using international Diabete Foundation and Adult Treatmen panel III criteria: associations with insulin resistance, disturbed body fat compartmentalization elevated c-reactive protein, and [corrected] hypoadiponectinemia. Diabetes Care. 2007;30(1):113-119. 
26. Young J, Rickenbach M, Weber R, et al. Body fat changes among antiretroviral-naïve patients on PI- and NNRTI-based HAART in the Swiss HIV Cohort Study. Antivir Ther. 2005;10(1):73-81.

27. Saint-Marc T, Partisani M, Poizot-Martin I, et al. A syndrome of peripheral fat wasting (lipodystrophy) in patients receiving long-term nucleoside analogue therapy. AIDS. 1999;13(13):1659-1667.

28. Mallon PW, Unemori P, Sedwell R, et al. In vivo, nucleoside reversetranscriptase inhibitors alter expression of both mitochondrial and lipid metabolism genes in the absence of depletion of mitochondrial DNA. $J$ Infect Dis. 2005;191(10):1686-1696.

29. Hammond E, Nolan D, James I, et al. Reduction of mitochondrial DNA content and respiratory chain activity accurs in adipocytes within 6-12 months of commencing nucleoside reverse transcriptase inhibitor therapy. AIDS. 2004;18(5):815-817.

30. Mercier S, Gueye NFN, Cournil A, et al. Lipodystrophy and metabolic disorders in HIV-1-infected adults on 4-to 9-year antiretroviral therapy in Senegal: a case control study. J Acquir Immune Defic Syndr. 2009;51(2):224-230.
31. Capeau J, Bastard JP, Caron M, et al. HIV, lipodystrophies and metabolic alterations. STV. 2003;15(9):487-498.

32. Capeau J, Valantin MA. Syndrome lipodystrophique au cours des traitements antirétroviraux. In: VIH de Girard PM, et al. (Eds.), Doin Paris, France. 2011; p. 528.

33. Nolan D. Do non-nucleoside reverse transcriptase inhibitors contribute to lipodystrophy? Drug Saf. 2005;28(12):1069-1074.

34. Lee GA, Seneviratne T, Noor MA, et al. The metabolic effects of lopinavir/ritonavir in HIV-negative men. AIDS. 2004;18(4): 641-649.

35. Mallon PM. Antiretroviral therapy-induced lipid alterations: in-vitro, animal and human studies. Curr Opin HIV AIDS . 2007;2(4):282-292.

36. Marceau G, Jacomet C, Ughetto S, et al. Survenue des syndromes lipodystrophiques et hyperlactatémiques Chez les patients infectés par le Virus de 1'Immunodéficience humaine. An Bio Clin. 2004;62(4):471478

37. Mulligan K, Parker RA, Komarow L, et al. Mixed patterns of changes in central and peripheral fat following initiation of antiretroviral therapy in a randomized trial. J Acquir Immune Defic Syndr. 2006;41(5):590-597. 